\title{
Mobile and Location Based Service using Augmented Reality: A Review
}

\author{
Prakash Kanade and Jai Prakash Prasad
}

\begin{abstract}
MAR (Mobile Augmented Reality) is delegated an innovation that gives similar capacities as AR (Augmented Reality), yet without the actual limitations of the area of an exploration office or testing region. A Location-Based Service (LBS) is an application for portable figuring which gives clients administrations dependent on their topographical area. Area based administrations are getting progressively famous with the ascent of cell phones with an ever increasing number of highlights (particularly Apple's iPhone and Android-based gadgets). Increasingly more substance is improved with geoinformation and can subsequently be seen in a virtual climate, however in real, portable conditions and in a setting delicate way fit to the requirements of the client. The definition and advantages of versatile increased reality and area based administrations and the mix of portable enlarged reality and area based administrations are broke down in this article. The issues are examined alongside the upsides and downsides.
\end{abstract}

Index Terms - Augmented Reality, LeenaBOT, Locationbased system, Mobile augmented reality.

\section{INTRODUCTION}

In these conditions, AR (Augmented Reality) presents itself as an exceptionally viable User Interface (UI) by consolidating visual information overlays with the actual world so clients can get an improved comprehension of their environmental factors. Over the most recent few years, the field of AR has developed quickly, being generally grasped in significant advertising lobbies for the vehicle business and in Hollywood.

AR (Augmented reality) furnishes setting mindful PC conditions with a particularly solid (UI). Computerized information is embedded into this present reality of an individual by AR frameworks with the goal that the person in question can decipher the data as happening in their current circumstance. MARS (Mobile augmented reality systems) offers this support of an extraordinarily fitted locale without restricting the whereabouts of the client.

In a perfect world, they work for all intents and purposes any place, any place required, giving a tangible layer of data to each climate. Through doing as such, they can reform the way where individuals are gone up against with information. PC introduced content is explicitly consolidated with the free-wandering individual's actual world, who can speak with it to see pertinent information, ask and address inquiries, and help out others [1]. The universe turns into the interface of the client.

AR (Augmented Reality) is a zone where different effective advancements and groundbreaking thoughts are

Submitted on February 12, 2021.

Published on March 18, 2021.

Prakash Kanade, Hobby Researcher in Robotics, Artificial Intelligence, IOT, USA.

(e-mail: Prakashsrs ${ }^{@}$ gmail.com) interconnected. MAR (Mobile Augmented Reality) is named an innovation that gives similar abilities as AR (Augmented Reality), yet without the actual requirements of the area of an exploration office or testing territory. To take a picture of the world and to surmise what computerized data applies to the current setting, Mobile AR uses various sensors. There are many observing strategies, going from GPS, GSM or cell LAN-based enormous scope answers for more exact attractive field-based arrangements, inertial arrangements (accelerometers and gyrators), sensors (for example radio recurrence ID), visual markers, or less following markers. GPS, for example, is valuable for significant distance synchronization of AR material, yet in addition excessively temperamental for short distances $(<45 \mathrm{~m})$. In any type of setting, the different arrangements might be pretty much utilized, however the exactness and scope of utilization got changes [2]. The AR machine will gather, with the guide of certain innovation, for instance, the situation of the client, what she is taking a gander at, and where and how rapidly she is moving [3].

A Location-Based Service (LBS) is an application for portable registering which furnishes clients with administrations dependent on their geological area.

This paper subsequently presents MAR from various perspectives, starting with a synopsis of the meaning of MAR as a rule, substance of MAR, and benefits. It is joined by area based assistance ideas, engineering, area based help preparing and executions. It likewise investigates the joining of Mobile Augmented Reality and Location Related Services. At last, alongside the difficulties [4], they closed.

\section{Augmented Reality}

AR (Augmented Reality) is a region where assorted effective advancements and novel thoughts are interconnected. AR is a multifaceted articulation that may apply to,

- An innovation or an innovation local area, a cross section innovation that utilizes numerous different advancements also,

- A definition that characterizes a future PC see,

- A logical territory of various fields,

- A computerized data medium and gui, and as of late additionally a discussion for the advancement of novel administrations and administrations Company.

Dr Jai Prakash Prasad, Professor, Don Bosco Institute of Technology, Bangalore, India

(e-mail: Jaiaasu@gmail.com) 


\section{Mobile Augmented ReAlity}

As a result of the quick advancement of electronic gadgets, AR is currently entering the versatile domain. From that point onward, installed cameras, detecting frameworks, for example, GPS and direction sensors, high-goal fullshading shows, rapid systems administration, high handling power, devoted 3D illustrations processors, and so forth were fitted with advanced mobile phones.

Regarding sensor innovations, for instance, cell phones can go about as outer eyes and ears to detect shrouded data in the surrounding climate. A skillful structure for creating AR applications and administrations [5] offers such a huge number of potential outcomes that are joined into one framework that is broadly scattered.

In any case, as an equipment, versatile AR isn't just about making a portable or hand-held PC. Rather than being bound to fixed areas and deliberately molded settings, for example, in clinical or modern applications, it is about AR being took into consideration really adaptable and inescapable settings and practices. Versatile AR settings and occasions could incorporate, for instance, 'in the wild' information journey, way discovering, administration and item assortment, interpersonal interaction, amusement and disclosure of more extensive regions. In correlation, military applications and fixes may utilize both handheld and fixed AR [6], for instance.

Six parts needed to give genuine MAR [7] were characterized in this work:

a. A virtual stage for the recovery of all connected data and the estimation of the perception of AR objects showed on the show.

b. Show to give the client recreated objects.

c. Atmosphere Registering. Camera criticism and head direction enlistment permits to introduce the AR protests precisely connected with this present reality.

d. To permit a portable individual to work and speak with others, wearable input and connection advancements

e. Remote systems administration with different elements and focal information bases for moment contact

f. Information assortment and access framework to supply the client with all proper natural foundation information expected for expansion.

\section{AugMENTED REALITY TYPES}

Marker-based AR: Marker-based AR in reality utilizes a marker or a static picture that fills in as an impetus for the upgraded insight. At the point when it is recognized [2][3], the maker will situate some computerized material over or close to the marker.

Marker-less AR: Marker-less AR works by filtering its general surroundings and permits the client to figure out where the reenacted item ought to be mounted. It relies upon the equipment of the unit, for example, the camera, GPS, advanced compass, and so forth.

Area based AR: In contrast with the past two types of AR, area based applications use GPS and the framework's computerized compass to decide the client's gadget area just as its area. Where the area of a client meets the default position, the reenacted object is appeared on the gadget. An area based AR model is Pokemon Go.

Superimposition AR: Superimposition AR considers a substance in reality and modifies the point of view of an article either somewhat or completely.

AR dependent on projection: AR dependent on projection is particular from different types of expanded reality without markers. It is utilized to make 3D models that can be interfaced with by the client. A projection-based AR can be utilized to show an example of another gadget or it's inside capacity.

\section{LITERATURE REVIEW}

Klinker et al. [8] show the gadget engineering of a MARS model for atomic force plant support use. For conditions including "x-beam vision," a capacity to see through hefty obstructions, AR is all around adjusted. AR may help development laborers who are endeavoring to locate a messed up link connect inside the dividers of a house, or the area of a spilled pipe under the outside of a street, by utilizing direct overlays of disguised framework. The exact area might have been consequently detected (for example by sensors introduced), in which case direct representation of the issue region by AR might be the quickest method to guide the consideration of the specialist to the right region. Then again, AR would be utilized as a steady technique in any case to evaluate the issue, in that it could envision any information that the laborer could acquire quickly and straightforwardly, testing the region with various sensors.

Feiner et al. [9] this model MARS gives an interface to a registry of eateries in New York City's Morningside Heights. Café data is given either with a 3D outline map, so the client can be coordinated to a specific area of their picking, or as away from of their preferred genuine area areas of cafés themselves. In the event that a foundation has been picked, the client can open a spring up window containing extra data about it: a short review, address and phone number, an inside outline and, available at the snap of a catch, a menu and, if suitable, criticism of the café and its outer website page.

Nusser et al. [10], Field spatial work. In geology and territorial sciences, field laborers may utilize AR methods to incorporate, think about and update study information and measurements in the field. An AR system could permit the disclosure of patterns in the field, not simply the lab, by advancing information assortment and show. It will be conceivable to check and match data promptly with records on paper.

Pavlik et al. [11] Journalism is another field where the utilization of remote media for a versatile writer who reports and tracks an arising report on the run could significantly affect MARS. For other working together writers and picture takers, MARS might be utilized to leave notes on the scene to decipher and follow up on.

Thomas et al. [12] the execution of arranged movies additionally shows the capability of innovation for amusement purposes. Rather than introducing 3D film "rides" to watchers in specific reason amusement park theaters, for example, the well-known Terminator 2 show at Universal Studios, mimicked entertainers in enhancements scenes could one day possess the very roads of the 
amusement parks, drawing in AR furnished guests in emotional activity? A few specialists have tried different things with adjusting portable AR advances to gaming as a promising beginning toward this path.

Feiner et al. [13] Continuous 2D pointing is talked about by a significant number handheld info gadgets. In the handheld space, trackballs, track-cushions, and gyroscopic mice can execute pointing capacities, the area of mice in work area gadgets, all of which hand-off information remotely to the host PC. In any case, it ought to be noticed that these gadgets are generally well known on the grounds that numerous analysts right now run basic WIMP UIs on their cell phone and wearable stages, missing a superior versatile UI standard. Exact $2 \mathrm{D}$ pointing represents an incredible danger to the engine capacities of a cell phone client. In any case, to screen cursor-less AR UIs, 2D pointing gadgets may likewise be utilized.

Thomas et al. [14] Different frameworks are more wasteful for brief easygoing use yet have been effectively utilized in examination models. For instance, utilizing such changed innovation as electrical touch cushions, flex sensors, accelerometers, and even power criticism frameworks, glove-based information instruments may distinguish hand developments, yet have the drawback of seeming cumbersome and limiting hands from being utilized in certifiable assignments. By and by, for certain MARS plans, the sturdiness and flexibility of glove developments have made the machine glove an info gadget of decision.

Starner et al. [15] In the opposite side, explore the recognizable proof of vision-based hand developments, which keeps the hands unhampered, however requests that a camera be worn on a cap or glasses, highlighting the area before the body of the individual, where hand motions are for the most part made.

Maia et al. [16] Normally, LBG maps genuine conditions into a virtual world, where players need to head out into genuine to explore the virtual world and perform exercises pertinent to the game itself. Maps, exercises, and other substance appeared on cell phones are customized to the player's position. Players can assemble virtual things, for example, weapons, ammo, or gems, as per the game objectives. In the virtual universe, or even in the actual world, these items might be utilized to acquire focal points. Geocaching, Ingress, and Pokémon Go are a few instances of regular LBG.

Raper et al. [17] Early LBS applications, communicated information prevalently to clients on cell phones through visual (for example maps) and hear-able (for example verbal directions) interfaces. Ongoing years have seen fast improvement towards more 'ordinary' and non-meddling LBS UIs, particularly on features of portrayal modes (for example visual, hear-able, and material), interface frameworks and gadgets (for example cell phones and smart watches), methodology of association (for example contact, movement, and look based), and information on foundation.

Majchrzak et al. [18] the estimation of LBS can be done in the research facility or in the field. Lab tests have the reward of being performed simpler and less expensive than concentrates in certifiable settings under directed conditions. This is on the grounds that the real biological systems where LBS advancements will at last be utilized are more convoluted, various, and unpredictable, at the danger of lacking natural legitimacy. Specialists have likewise demonstrated that individual's demonstration distinctively in the research facility comparative with the genuine one. To investigate the positives and detriments by utilizing research facility and field-based methodologies in testing Pounds, just as how to all the more likely coordinate them, further examination should be directed.

Azuma et al. [19] an exhaustive investigation of the execution fields of LBS was given by. They demonstrated that the dominant part classification of LBS applications were versatile aides and route frameworks (for example vehicle route frameworks and person on foot route frameworks). Portable aides might be seen as intelligent advisers for the client's reality that are conservative, area delicate and data rich. They likewise have highlights for the travel industry and relaxation utilizes, for example, cell phone search, 'youare-here' diagrams, and local area experts. Route frameworks are intended to help individuals discover exercises in obscure conditions, for example, for vehicle drivers or people on foot, as found in Fig. 1. Albeit a portion of the significant LBS applications keep on being cell phone aides and route frameworks are as yet being improved (for example milestone based route and consideration of constant traffic data).

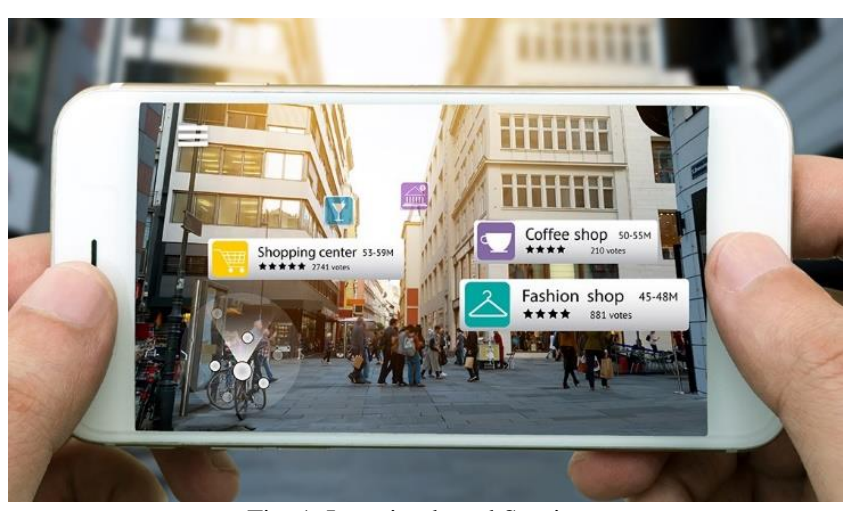

Fig. 1. Location based Services.

A few judgment factors must be weighed when picking a head-worn screen for cell phone AR. The option between optical transparent and video transparent showcases is perhaps the most petulant subjects inside the AR science local area. The manner in which solution focal points or shades are optical transparent screens are clear. To layer the PC produced picture on top of the client's perspective on the world, they utilize optical combiners, for example, reflect bar splitters. An image taken from such glasses is found in Fig. 2 (a). Video sees, conversely, give a more backhanded, separated impression of the world through showcases. Simply before the client's eyes, a couple of smaller than usual camcorders, mounted on the head-worn screen, record video floods of the world before the client that are projected on non-straightforward presentations with adequate optics. Until it's shipped off the glasses to make AR overlays, the machine will change the video picture. Fig. 2 shows a model (b). 


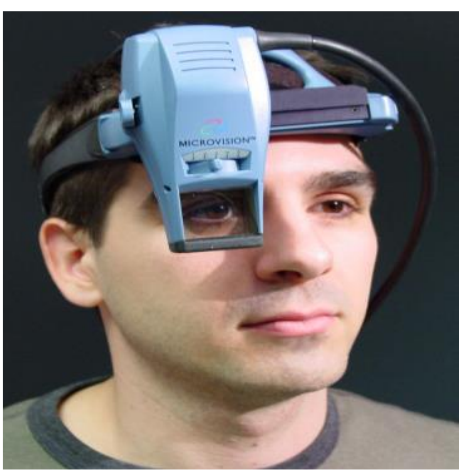

(a)

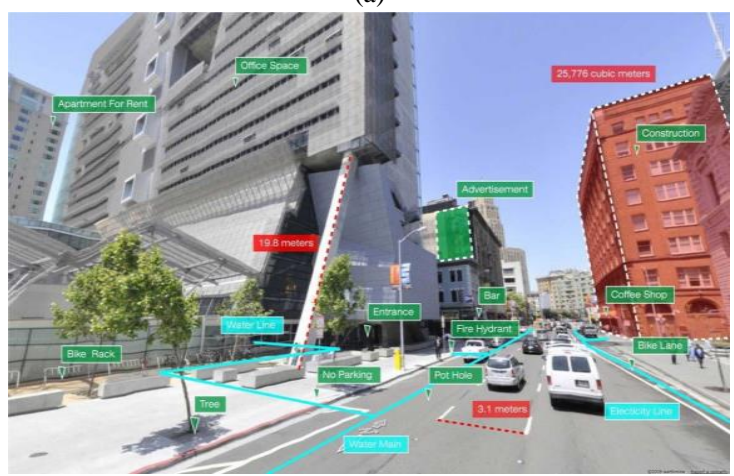

(b)

Fig. 2. (a) Optical see-through and (b)video see through AR implementation

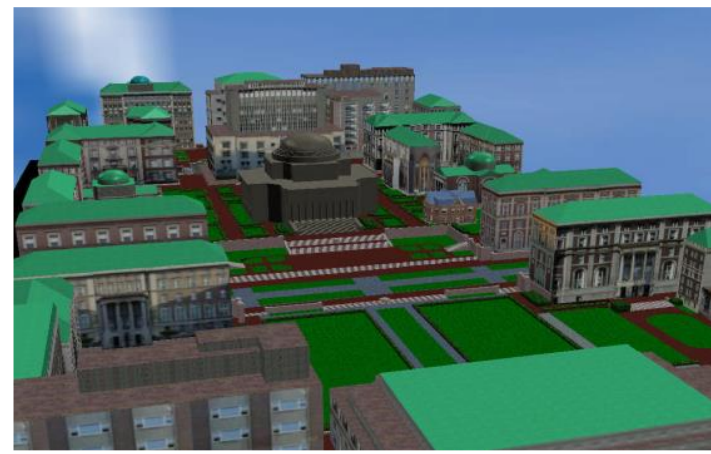

(a)

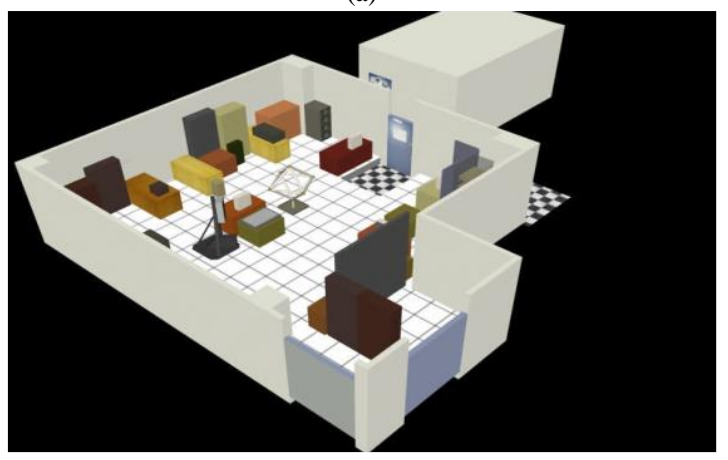

(b)

Fig. 3. Environmental modelling: (a) Model of a campus; (b) Model of a laboratory.

A portrayal of the world shouldn't be photograph sensible for the reasons depicted up until this point. Materials, designs, and maybe even mathematical intricacy might be ignored. Truly, at times just a "cloud" of detached 3D example focuses is utilized in model-based observing. For comment purposes, or to give a layout of the genuine setting, more sensible mathematical portrayals of true develops, for example, those appeared in Fig 3 , are likewise utilized. For instance, a structure that is covered from the perspective on the client can be seen by AR in its careful concealed position, permitting the client to see through dividers in some sense [20].

Wikitude was dispatched on the iPhone and Symbian stages on Android in 2008 and later on. It was named the best AR program of 2010 by Augmented Earth perusers. This gathers data and overlays the geo-found subtleties on the PC from Wikipedia and Qype. It accompanies a ton of universes that can be superimposed over this present reality that can be seen through the camera of the cell phone. The clients themselves and area based computerized content that can be gotten to through the Wikitude programming structure [24] can fabricate POIs.

For iOS cell phones, ARKit is Apple's increased reality advancement device. ARKit permits designers to make iPad and iPhone AR encounters in high goal. Computerized 3D content, articles, and characters can be upheld in gadget caught conditions. AR scenes made by a solitary individual are endless, and people visiting the site will later access them. Movement coprocessors, for example, the iPhone 7 Plus M10 can show spinner, accelerometer, and magnetometer information productively. Pokémon Go utilizations ARKit [25] to have a smoother experience.

The Vuforia stage fuses best in class, steady and effective electronic picture acknowledgment and gives the portable client numerous capacities. A camera that takes picture casings and moves substance to a tracker is a Vuforia SDKbased AR-App; a camera-based picture converter in an OpenGL ES delivering design; a tracker that can simultaneously stack and actuate a few informational collections and incorporate certifiable antiquities followed and recognized by $\mathrm{PC}$ vision calculations. It likewise furnishes quicker neighborhood target following the capacity to plan five targets all the while. The SDK underpins both local iOS and Android innovations, while likewise permitting the advancement of Unity AR applications that can be immediately ported to the two stages [26].

Kuitunen et al. [27] is an open-source versatile application structure which permits engineers, alongside local stage capacities, to fabricate genuine and energizing portable applications dependent on JavaScript and React. ReactJS, a Facebook library for making UIs, depends on it. UIs can be made on the two iOS and Android gadgets with the React Native Framework. It will be agreeable with different frameworks remembering Windows and TvOS for the not so distant future. The improvement of the React Native [9] system is moderately brisk, speedier, and more viable and has lower advancement costs. These segments can be reused (simply code once) in creating both Android and iOS applications on the grounds that React Native segments have partner advantages, which assists with saving advancement time, i.e., helps in the turn of events and reuse of Seamless cross-stage and code. Utilizing JavaScript, after a code update is finished, it emphasizes the local forms at a lighting pace when the code is saved.

For designers to effortlessly make local cross-stage AR/VR applications utilizing the ReactNative Framework, ViroReact is an open source engineer stage. It upholds ARKit just as ARCore which can be joined with the most recent application. This stage is included transcendently of two key parts. Every one of them is a superior local 3D 
delivering motor working on local strings that utilizes the gadget's graphical equipment chip. Slack free is this delivering motor yet upgraded 3D documents and in a perfect world low-poly records for cell phones should be utilized. The 3D record organizes that are upheld are .obj, .fbx and .gltf documents. A custom expansion of React for $\mathrm{AR}$ and VR creation is the other part. In ViroReact, coding should be done once, and both cell phone AR (ARKit, ARCore) and VR (Cardboard iOS/Android, GearVR, Daydream) will utilize this code. It is anything but difficult to see the fabricated application with the guide of a free Viro Testbed application and to see the enhancements made effectively, i.e., it helps in Rapid Iteration. Utilizing the explanatory markup language makes it a simple to-learn apparatus and it is along these lines not imperative to utilize Xcode or Android Workshop. In the event that future VR highlights are to be utilized in the application, the designer doesn't have to fabricate another API, in light of the fact that similar API utilized for AR can be reused in Viro React.

\section{CONCLUSION}

We gave an outline of the field of versatile AR in this section, including verifiable patterns, potential for the future, zones of utilization, troubles, segments, and details, best in class frameworks, and standards of UI. As exemplified by the rising number of scholarly commitments and business propels since the mid-1990s, AR and wearable registering are progressively developing zones. In the movement towards versatile $\mathrm{AR}$, we have arrived at a huge point in that the innovation accessible is sufficient for a developing number of stunning exploration models, yet not yet enough effective, general,, and agreeable for mass acknowledgment. It will require some investment for versatile AR to enter the standard of registering comparative with different applications referenced in this book, which are right away feasible utilizing the present innovations. At the point when it gets pervasive, however, portable AR can have a monstrous effect.

In the territories of PC equipment scaling down, battery configuration, show innovation, sensor innovation, exactness and strength of checking, general vision-based following and scene mindfulness, and by and large solace, we anticipate greater headway. For setting based figuring when all is said in done, and AR specifically, we anticipate the appearance of dispersed information frameworks, adding to far and away superior information access capacities for cell phone gadgets [28]. At long last, we anticipate that, without losing protection and accommodation, the upsides of versatile AR can be cultivated. Exploration and headway of a zone that may profoundly affect social frameworks and standards ought to be trailed by close investigation of how the admirable aspects of our social harmony can be kept up and improved. AR has a wide assortment of different fields as an innovation. Applications intended to offer the client a virtual perspective on the rooms, simple appointments for flights and customized ongoing subtleties, especially in the movement and the travel industry enterprises.

\section{REFERENCES}

[1] Prakash Kanade, Monis Akhtar, Fortune David, "Remote Monitoring Technology for COVID-19 Patients" European Journal of Electrical Engineering and Computer Science, vol. 5, no. 1, Pages. 44-47, 2021.

[2] Prakash Kanade, Monis Akhtar, Fortune David, "Computer Networking and Technology Improvement in the Age of COVID-19" International Journal of Advanced Networking and Applications (IJANA), vol. 12, no. 03, Pages. 4592-4595, 2020.

[3] Thomas Olsson, "User Expectations and Experiences of Mobile Augmented Reality Services" Tampere University of Technology Tampere 2012, ISBN 978-952-15-2931-3.

[4] Azuma, R. T., A survey of augmented reality, Presence: Tele operators \& Virtual Environments, 6(4), 355-385, 1997.

[5] Poetker, B., What Is Augmented Reality? Most Common Types of AR Used Today. Learning Hub, 2019. https://learn.g2.com/augmentedreality.

[6] Gatis, Z., Marker-based vs marker less augmented reality: pros, cons \& examples, AR insights, 2019. https://overlyapp.com/blog/markerbasedvs-markerless-augmented-reality-pros-cons.

[7] Lypchenko, S., How to Build a Location-Based AR Application. AR Post, 2019. https://arpost.co /2019/10/29/ how-to-build-alocationbased-ar-application.

[8] Castro, P., Chiu, P., Kremenek, T., and Muntz, R., A probabilistic room location service for wireless networked environments. In Proc ACM UbiComp 2001: Ubiquitous Computing, volume 2201 of Lecture Notes in Computer Science, pages 18-35, 2001.

[9] Cometa Networks, Wholesale nationwide broadband wireless internet access, 2002. http://www.cometanetworks. Com.

[10] Klinker, G., Creighton, O., Dutoit, A. H., Kobylinski, R., Vilsmeier, C., and Brügge, B., Augmented maintenance of power plants: a prototyping case study of a mobile AR system. In Proc. ISAR '01 (IEEE and ACM Int. Symposium on Augmented Reality), pages 124133, 2001, New York, NY.

[11] Feiner, S., Augmented Reality: A new way of seeing. Scientific American, 286(4):48-55, 2002.

[12] Nusser, S. M., Miller, L. L., Clarke, K., and Goodchild, M. F. Geospatial IT for mobile field data collection. Communications of the ACM, 46(1):63-64, 2003

[13] Pavlik, J. V., Journalism and New Media. Columbia University Press, New York, NY, 2001

[14] Thomas, B., Close, B., Donoghue, J., Squires, J., De Bondi, P., Morris M., and Piekarski, W., ARQuake: An outdoor/indoor augmented reality first person application. In Proc. ISWC '00 (Fourth Int. Symp. on Wearable Computers), pages 139-146, 2000, Atlanta, GA.

[15] Feiner, S., MacIntyre, B., Höllerer, T., and Webster, A., A touring machine: Prototyping 3D mobile augmented reality systems for exploring the urban environment. In Proc. ISWC '97 (First IEEE Int. Symp. on Wearable Computers), pages 74-81, 1997, Cambridge, MA.

[16] Thomas, B. H. and Piekarski, W., Glove based user interaction techniques for augmented reality in an outdoor environment. Virtual Reality: Research, Development, and Applications, 6(3):167-180, 2002. Springer-Verlag London Ltd.

[17] Starner, T., Weaver, J., and Pentland, A., A wearable computing based American Sign Language recognizer. In Proc. ISWC '97 (First Int. Symp. on Wearable Computers), pages 130-137, 1997b, Cambridge, MA.

[18] Maia, L. F., C. Nolêto, M. Lima, C. Ferreira, C. Marinho, W. Viana, and F. Trinta, "LAGARTO: A LocAtion Based Games AuthoRing Tool Enhanced with Augmented Reality Features." Entertainment Computing 22: 3-13. 2017, doi:10.1016/j.entcom.2017.05.001.

[19] Raper, J., G. Gartner, H. Karimi, and C. Rizos, "Applications of Location-Based Services: A Selected Review." Journal of Location $\begin{array}{lllll}\text { Based Services } 1 & \text { (2): } 89-111, \quad 2007 \mathrm{~b}, \quad \text { doi: }\end{array}$ $10.1080 / 17489720701862184$.

[20] Majchrzak, T., \& Grønli, T. M., Comprehensive analysis of innovative cross-platform app development frameworks. In Proceedings of the 50th Hawaii International Conference on System Sciences, 2017.

[21] Azuma, R. T., A survey of augmented reality. Presence: Tele operators \& Virtual Environments, 6(4), 355-385, 1997.

[22] J. Carmigniani, B. Furht, M. Anisetti, P. Ceravolo, E. Damiani, and M. Ivkovic, Augmented reality technologies, systems and applications. Multimed. Tools Appl. Vol. 51(1), pp. 341-377, 2010.

[23] M. Gervautz and D. Schmalstieg, Anywhere interfaces using handheld augmented reality. Computer. Vol. 45(7), pp. 26-31, 2012.

[24] S. A. Malik, and M. Azuddin, the Use of Technology by Older People. International Journal of Interactive Digital Media. Volume 1(3), pp. $65-58,2013$ 
[25] Engel, J., Schöps, T., \& Cremers, D., LSD-SLAM: Large Scale Direct Monocular SLAM. European Conference on Computer Vision-ECCV 2014. (pp. 834-849), 2014. Springer International Publishing.

[26] https://www.wikitude.com.

[27] https://developer.apple.com/documentation/arkit.

[28] Prakash Kanade, Sunay Kanade, "Medical Assistant Robot ARM for COVID-19 Patients Treatment - A Raspberry Pi Project," International Research Journal of Engineering and Technology (IRJET), vol. 7, no. 10, Pages. 105-111, 2020. 